TRANSACTIONS OF THE

AMERICAN MATHEMATICAL SOCIETY

Volume 361, Number 6, June 2009, Pages 3343-3357

S 0002-9947(08)04713-2

Article electronically published on December 31, 2008

\title{
BI-INVARIANT METRICS ON THE GROUP OF SYMPLECTOMORPHISMS
}

\author{
ZHIGANG HAN
}

\begin{abstract}
This paper studies the extension of the Hofer metric and general Finsler metrics on the Hamiltonian symplectomorphism group $\operatorname{Ham}(M, \omega)$ to the identity component $\operatorname{Symp}_{0}(M, \omega)$ of the symplectomorphism group. In particular, we prove that the Hofer metric on $\operatorname{Ham}(M, \omega)$ does not extend to a bi-invariant metric on $\operatorname{Symp}_{0}(M, \omega)$ for many symplectic manifolds. We also show that for the torus $\mathbb{T}^{2 n}$ with the standard symplectic form $\omega$, no Finsler metric on $\operatorname{Ham}\left(\mathbb{T}^{2 n}, \omega\right)$ that satisfies a strong form of the invariance condition can extend to a bi-invariant metric on $\operatorname{Symp}_{0}\left(\mathbb{T}^{2 n}, \omega\right)$. Another interesting result is that there exists no $C^{1}$-continuous bi-invariant metric on $\operatorname{Symp}_{0}\left(\mathbb{T}^{2 n}, \omega\right)$.
\end{abstract}

\section{INTRODUCTION AND MAIN RESULTS}

1.1. Results on the Hofer metric. The Hofer metric is a bi-invariant metric defined on the Hamiltonian diffeomorphism $\operatorname{group} \operatorname{Ham}(M, \omega)$, and it is natural to consider its possible extension to the identity component $\operatorname{Symp}_{0}(M, \omega)$ of the symplectomorphism group $\operatorname{Symp}(M, \omega)$. In fact, Banyaga and Donato 2 constructed such an extension for certain symplectic manifolds, but the resulting metric is only right-invariant on $\operatorname{Symp}_{0}(M, \omega)$. The purpose of this paper is to exhibit some obstruction for the Hofer metric and other bi-invariant metrics to extend from $\operatorname{Ham}(M, \omega)$ to $\operatorname{Symp}_{0}(M, \omega)$. Here $(M, \omega)$ is a closed symplectic manifold with symplectic form $\omega$.

It will be useful to consider the following concept of bounded symplectomorphisms introduced by Lalonde and Polterovich in 8 . More precisely, let $\rho$ be the Hofer norm, i.e. $\rho(f)$ is the Hofer distance between $i d$ and $f$ for all $f \in \operatorname{Ham}(M, \omega)$. For each $\phi \in \operatorname{Symp}(M, \omega)$, define

$$
r(\phi):=\sup \{\rho([\phi, f]) \mid f \in \operatorname{Ham}(M, \omega)\},
$$

where $[\phi, f]:=\phi f \phi^{-1} f^{-1}$ is the commutator of $\phi$ and $f$.

Definition 1.1. A symplectomorphism $\phi \in \operatorname{Symp}(M, \omega)$ is said to be bounded if $r(\phi)<\infty$, and unbounded otherwise.

Denote by $\operatorname{BI}_{0}(M, \omega)$ the set of all bounded symplectomorphisms in $\operatorname{Symp}_{0}(M, \omega)$. The inequality $\rho([\phi, f]) \leqslant 2 \rho(\phi)$ implies that $\operatorname{Ham}(M, \omega)$ is a subgroup of $\mathrm{BI}_{0}(M, \omega)$. The converse is the following conjecture in 8 .

Received by the editors October 1, 2007.

2000 Mathematics Subject Classification. Primary 53D35; Secondary 57R17.

Key words and phrases. Hofer metric, Finsler metric, bi-invariant extension, admissible lift.

(C)2008 American Mathematical Society

Reverts to public domain 28 years from publication 
Conjecture 1.2 (Bounded isometry conjecture). For all $(M, \omega), \mathrm{BI}_{0}(M, \omega)=$ $\operatorname{Ham}(M, \omega)$.

This conjecture was proved in 8 for closed surfaces with area form and for arbitrary products of closed surfaces of genus greater than 0 with product symplectic form. Lalonde and Pestieau [9] give a positive answer for product symplectic manifolds $M=N \times W$ with $N$ any product of closed surfaces and $W$ any closed symplectic manifold of the first real Betti number equal to zero. Also, it was recently confirmed by Han [5] for the Kodaira-Thurston manifold with the standard symplectic form and for the 4-torus with all linear symplectic forms. The proofs in [8] and [5] use the energy-capacity inequality (cf. Lalonde and McDuff [7]) on the universal cover $\widetilde{M}$ of $M$, while the stable energy-capacity inequality is the main tool in 9 .

It turns out that the existence of unbounded symplectomorphisms serves as an obstruction to bi-invariant extensions of the Hofer metric to $\operatorname{Symp}_{0}(M, \omega)$. The following theorem follows almost directly from the definition of unbounded symplectomorphisms.

Theorem 1.3. Let $(M, \omega)$ be a closed symplectic manifold. Assume that there exists some $\phi \in \operatorname{Symp}_{0}(M, \omega)$ which is unbounded in the sense of Definition 1.1. Then the Hofer metric does not extend to a bi-invariant metric on $\operatorname{Symp}_{0}(M, \omega)$.

Proof. Assume the Hofer metric $\rho$ extends to a bi-invariant metric on $\operatorname{Symp}_{0}(M, \omega)$, which we still denote by $\rho$. Then given $\phi \in \operatorname{Symp}_{0}(M, \omega)$, using the triangle inequality and the fact that $\rho$ is bi-invariant and $\rho\left(\phi^{-1}\right)=\rho(\phi)$, we have

$$
\rho([\phi, f])=\rho\left(\phi f \phi^{-1} f^{-1}\right) \leqslant \rho(\phi)+\rho\left(f \phi^{-1} f^{-1}\right)=\rho(\phi)+\rho\left(\phi^{-1}\right)=2 \rho(\phi)
$$

for all $f \in \operatorname{Ham}(M, \omega)$. Taking the supremum over all $f \in \operatorname{Ham}(M, \omega)$ gives $r(\phi) \leqslant$ $2 \rho(\phi)<\infty$. It then follows from Definition 1.1 that all elements $\phi \in \operatorname{Symp}_{0}(M, \omega)$ are bounded, which contradicts our assumption. Hence the proof is completed.

In particular, the above theorem applies to all symplectic manifolds where the bounded isometry conjecture holds. However, it is in general difficult to prove the bounded isometry conjecture, since one has to show that all non-Hamiltonian symplectomorphisms are unbounded. On the other hand, it is often easier to find one single unbounded symplectomorphism, which is sufficient for our purposes. For instance, combining the above theorem with Theorem 1.4.A in [8], one immediately has the following

Corollary 1.4. Let $L \subset M$ be a closed Lagrangian submanifold admitting a Riemannian metric with non-positive sectional curvature, and whose inclusion in $M$ induces an injection on fundamental groups. If there exists some $\phi \in \operatorname{Symp}_{0}(M, \omega)$ such that $\phi(L) \cap L=\emptyset$, then the Hofer metric does not extend to a bi-invariant metric on $\operatorname{Symp}_{0}(M, \omega)$.

Proof. By Theorem 1.4.A in 8, such $\phi$ must be unbounded. The corollary follows from Theorem 1.3

Thus, we have the following conjecture which seems more accessible than the bounded isometry conjecture.

Conjecture 1.5. For all symplectic manifolds $(M, \omega)$ such that $\operatorname{Symp}_{0}(M, \omega)$ is not identical to $\operatorname{Ham}(M, \omega)$, the Hofer metric on $\operatorname{Ham}(M, \omega)$ does not extend to a bi-invariant metric on $\operatorname{Symp}_{0}(M, \omega)$. 
Remark 1.6. Besides all manifolds mentioned above, this conjecture also holds for $M=\Sigma \times W$ with $\Sigma$ any closed surface of positive genus and $W$ any closed symplectic manifold. One can simply argue, using the stable energy-capacity inequality as in Lalonde and Pestieau [9], that $\phi \times i d \in \operatorname{Symp}_{0}(M)$ is unbounded with $\phi \in \operatorname{Symp}_{0}(\Sigma)$ any non-Hamiltonian symplectomorphism and $i d$ the identity map on $W$. Note that we are able to drop the assumption on $W$ from Lalonde and Pestieau's result (cf. Theorem 1.3 of [9]) simply because the bounded isometry conjecture is a stronger statement than our conjecture.

1.2. Results on other bi-invariant Finsler metrics. In this subsection, we consider general Finsler metrics on $\operatorname{Ham}(M, \omega)$. In order to formulate our main result, we shall need some preliminaries. Let $G$ be an $\infty$-dimensional Fréchet Lie group; for our purposes, it is either $\operatorname{Ham}(M, \omega)$ or $\operatorname{Symp}_{0}(M, \omega)$. Let $d$ be a biinvariant distance function on $G$. Define $\rho$ to be the function on $G$ such that for all $f \in G$,

$$
\rho(f):=d(i d, f) \text {. }
$$

The following properties of $\rho$ follow directly from the properties of the bi-invariant distance function $d$ :

(a) $\rho(f) \geqslant 0$, and $\rho(f)=0 \Rightarrow f=i d$.

(b) $\rho(f)=\rho\left(f^{-1}\right)$.

(c) $\rho(f g) \leqslant \rho(f)+\rho(g)$.

(d) $\rho\left(f g f^{-1}\right)=\rho(g)$.

Such a function $\rho$ is called a bi-invariant norm 1 If $\rho$ satisfies all the above properties except (a), then $\rho$ is called a bi-invariant pseudo-norm. For our purposes, it is sometimes more convenient to deal with a bi-invariant norm $\rho$ than to deal with a bi-invariant distance function $d$, although they are essentially equivalent.

Now we recall the concept of Finsler metrics on $\operatorname{Ham}(M, \omega)$. Let $(M, \omega)$ be a closed symplectic manifold of dimension $2 n$. Denote by $\mathcal{A}$ the space of all normalized smooth functions on $M$, i.e. all smooth functions $F$ on $M$ such that $\int_{M} F \omega^{n}=0$. It is well known that $\mathcal{A}$ can be identified with the space of all Hamiltonian vector fields, which is the Lie algebra 22 of the $\infty$-dimensional Lie group $\operatorname{Ham}(M, \omega)$.

Since all tangent spaces to the group $\operatorname{Ham}(M, \omega)$ are identified with $\mathcal{A}$, every choice of norm $\|\cdot\|$ on $\mathcal{A}$ gives rise to a pseudo-distance on $\operatorname{Ham}(M, \omega)$ in the following way. We define the length of a smooth Hamiltonian path $\alpha:[0,1] \rightarrow$ $\operatorname{Ham}(M, \omega)$ as

$$
\text { length }(\alpha):=\int_{0}^{1}\|\dot{\alpha}(t)\| d t=\int_{0}^{1}\left\|F_{t}\right\| d t,
$$

where $F_{t}(x)=F(t, x)$ is the time-dependent Hamiltonian function generating the path $\alpha$. This is the usual notion of Finsler length. The distance between two Hamiltonian symplectomorphisms $f$ and $g$ is defined by

$$
d(f, g):=\inf \{\operatorname{length}(\alpha)\},
$$

\footnotetext{
${ }^{1}$ In view of property (d), it might be more consistent to call $\rho$ a conjugate-invariant norm. However, we shall call it bi-invariant to emphasize that the corresponding distance function is bi-invariant. We shall sometimes also refer to $\rho$ as a bi-invariant metric.

${ }^{2} \mathrm{As}$ a vector space, the Lie algebra is by definition the tangent space to the Lie group at the identity. The tangent spaces to the Lie group at other points are identified with the Lie algebra with the help of right shifts of the group.
} 
where the infimum is taken over all Hamiltonian paths $\alpha$ connecting $f$ and $g$. It is easy to verify that $d$ is a pseudo-distance function. Denote by $\rho(f)$ the distance between identity and $f$, i.e.

$$
\rho(f):=d(i d, f) .
$$

Then $\rho$ is a pseudo-norm. Such a pseudo-norm is called a Finsler pseudo-norm, and the induced pseudo-metric is called a Finsler pseudo-metric.

The adjoint action of the Lie group $\operatorname{Ham}(M, \omega)$ on its Lie algebra $\mathcal{A}$ is the standard action of diffeomorphisms on functions, i.e. $A d_{f} G=G \circ f^{-1}$ for all $G \in \mathcal{A}$ and $f \in \operatorname{Ham}(M, \omega)$. We say a norm $\|\cdot\|$ on $\mathcal{A}$ is $\operatorname{Ham}(M, \omega)$-invariant if $\|\cdot\|$ is invariant under the adjoint action of $\operatorname{Ham}(M, \omega)$, i.e. $\left\|G \circ f^{-1}\right\|=\|G\|$ for all $G \in \mathcal{A}$ and $f \in \operatorname{Ham}(M, \omega)$. Note that the fact that $\|\cdot\|$ is $\operatorname{Ham}(M, \omega)$-invariant implies that the induced Finsler pseudo-metric is bi-invariant.

It is highly non-trivial to check whether such a pseudo-metric $\rho$ is non-degenerate. When it is, $\rho$ will be called a Finsler metric, and $\rho(f)$ will be referred to as the Finsler norm of $f$. On one hand, it is now well known that the $L_{\infty}$ norm on $\mathcal{A}$ gives rise to the Hofer metric on $\operatorname{Ham}(M, \omega)$, which is non-degenerate for all $(M, \omega)$. This was discovered and proved by Hofer [6] for the case of $\mathbb{R}^{2 n}$, then generalized by Polterovich [13. to some larger class of symplectic manifolds, and finally proved in the full generality by Lalonde and McDuff in [7. Note that the Hofer metric is bi-invariant. On the other hand, Eliashberg and Polterovich showed in 3$]$ that for $1 \leqslant p<\infty$, the Finsler pseudo-metric on $\operatorname{Ham}(M, \omega)$ induced by the $L_{p}$ norm on $\mathcal{A}$ vanishes identically. Thus the following question arises in [3] and [14].

Question 1.7. Which invariant norms on $\mathcal{A}$ give rise to genuine bi-invariant Finsler metrics? Is it true that such norms are always bounded below by $C\|\cdot\|_{\infty}$ ?

This question was studied by Ostrover and Wagner in [12]. One of their main results is the following

Theorem 1.8 ([12, Theorem 1.3]). Let $\|\cdot\|$ be a $\operatorname{Ham}(M, \omega)$-invariant norm on $\mathcal{A}$ such that $\|\cdot\| \leqslant C\|\cdot\|_{\infty}$ for some constant $C$, but the two norms are not equivalent. Then the associated pseudo-distance function on $\operatorname{Ham}(M, \omega)$ vanishes identically.

In general, Question 1.7 is still open, although the above theorem seems to imply that the answer to the second question is very likely to be positive. If this was the case, it would imply that all bi-invariant Finsler metrics are bounded below by a constant multiple of the Hofer metric. Therefore all the non-extension results above concerning the Hofer metric would still be valid for any bi-invariant Finsler metric on $\operatorname{Ham}(M, \omega)$. However, since all these are not yet completely understood, we find it interesting to have some kind of non-extension result for general Finsler metrics. In particular, we consider Finsler metrics induced by $\chi$-invariant norms which we shall define below.

Definition 1.9. Let $H$ be a normalized Hamitonian function in $\mathcal{A}$. We define the characteristic $\chi_{H}$ of $H$ to be the function from $(-\infty,+\infty)$ to $[0,1]$ such that

$$
\chi_{H}(c):=\frac{\operatorname{vol}(\{p \in M \mid H(p)<c\}, \omega)}{\operatorname{vol}(M, \omega)} .
$$

Definition 1.10. For $F, G \in \mathcal{A}$, we say $F$ is $\chi$-equivalent to $G$ if $\chi_{F}=\chi_{G}$.

For instance, if $F=G \circ \phi$ for some volume preserving diffeomorphism $\phi$, then $F$ and $G$ are $\chi$-equivalent. Also, let $\pi: \mathbb{T}^{2 n} \rightarrow \mathbb{T}^{2 n}$ be a covering map of $\mathbb{T}^{2 n}$ over 
itself, and let $H$ be a smooth Hamiltonian function on $\mathbb{T}^{2 n}$. Then $H$ is $\chi$-equivalent to $H \circ \pi$.

Definition 1.11. A norm $\|\cdot\|$ on $\mathcal{A}$ is said to be $\chi$-invariant if all $\chi$-equivalent Hamiltonian functions have the same norm, i.e. $\|F\|=\|G\|$ if $\chi_{F}=\chi_{G}$.

For example, $L_{p}$ norm and $L_{\infty}$ norm are $\chi$-invariant. Observe that a $\chi$-invariant norm $\|\cdot\|$ on $\mathcal{A}$ is necessarily $\operatorname{Ham}(M, \omega)$-invariant. Hence the induced Finsler metric $\rho$ on $\operatorname{Ham}(M, \omega)$ must be bi-invariant. The following proposition, which follows from a result by Ostrover and Wagner [12, explains why the $\chi$-invariance condition on the norm $\|\cdot\|$ is a reasonable one.

Proposition 1.12. Any $\operatorname{Ham}(M, \omega)$-invariant norm $\|\cdot\|$ on $\mathcal{A}$ which is bounded from above by $\|\cdot\|_{\infty}$ is $\chi$-invariant.

Proof. It is proved in [12] (Theorem 1.4) that such a norm $\|\cdot\|$ can be extended to a (semi)norm $\|\cdot\| \leqslant C\|\cdot\|_{\infty}$ on $L_{\infty}(M)$ which is invariant under all measure preserving bijections on $M$.

Let $F, G \in \mathcal{A}$ such that $\chi_{F}=\chi_{G}$. Then there exist two sequences of step functions $F_{n}, G_{n} \in L_{\infty}(M)$ and a sequence of measure preserving bijections $\phi_{n}$ on $M$ such that for each $n, F_{n}$ coincides with $G_{n} \circ \phi_{n}$ except for a measure zero set, and $F_{n}$ and $G_{n}$ converge to $F$ and $G$ respectively in $L_{\infty}(M)$. Thus we get $\left\|F_{n}\right\|=\left\|G_{n} \circ \phi_{n}\right\|=\left\|G_{n}\right\|$, and since $\|\cdot\|$ is bounded from above by $\|\cdot\|_{\infty}$, we also have $\left\|F_{n}\right\| \rightarrow\|F\|$ and $\left\|G_{n}\right\| \rightarrow\|G\|$. Therefore we conclude that $\|F\|=\|G\|$, which completes the proof.

We are ready to state our main result.

Theorem 1.13. Let $\left(\mathbb{T}^{2 n}, \omega\right)$ be the torus with the standard symplectic form $\omega$, and let $\rho$ be a $\chi$-invariant Finsler metric on $\operatorname{Ham}\left(\mathbb{T}^{2 n}, \omega\right)$, i.e. a Finsler metric induced by a $\chi$-invariant norm $\|\cdot\|$. Then $\rho$ does not extend to a bi-invariant metric on $\operatorname{Symp}_{0}\left(\mathbb{T}^{2 n}, \omega\right)$.

Question 1.14. We shall see below in the proof of Theorem 1.13 that we are using the fact that the diameter of $\operatorname{Ham}\left(\mathbb{T}^{2 n}, \omega\right)$ with respect to any $\chi$-invariant Finsler metric $\rho$ is infinite. Actually, if the diameter with respect to $\rho$ is finite, one can always extend it bi-invariantly to $\operatorname{Symp}_{0}\left(\mathbb{T}^{2 n}, \omega\right)$ by giving a sufficiently large constant value for all non-Hamiltonian symplectomorphisms (see Remark 3.11). The question is, will the infiniteness of the diameter of $\operatorname{Ham}\left(\mathbb{T}^{2 n}, \omega\right)$ be sufficient to prove that $\rho$ does not extend to a bi-invariant metric on $\operatorname{Symp}_{0}\left(\mathbb{T}^{2 n}, \omega\right)$ ?

Organization of the paper. Section 2 is devoted to the proof of Theorem 1.13 and a discussion on the possible generalizations. In Section 3 we present various results, including Theorem 3.6 which states that there exists no $C^{1}$-continuous bi-invariant metric on $\operatorname{Symp}_{0}\left(\mathbb{T}^{2 n}, \omega\right)$. We also construct bi-invariant metrics on $\operatorname{Symp}_{0}(M, \omega)$ and discuss their topological properties.

\section{Proof of Theorem 1.13 and generalizations}

In this section, we first formulate and prove Theorem 2.2. a generalization of Theorem 1.13 that works for any (not necessarily Finsler) bi-invariant metric, and then use it to prove Theorem 1.13 To begin with, we recall the concept of admissible lifts which was first introduced by Lalonde and Polterovich [8]. We shall point 
out that our definition is slightly different from theirs, but the two definitions are equivalent.

Let $\pi:(\widetilde{M}, \widetilde{\omega}) \rightarrow(M, \omega)$ be a symplectic covering map, i.e. a covering map between two symplectic manifolds such that $\widetilde{\omega}=\pi^{*} \omega$. For every $g \in \operatorname{Ham}(M, \omega)$, assume $g$ is the time- 1 map of the Hamiltonian flow generated by the time-dependent Hamiltonian function $H_{t}$. An admissible lift $\widetilde{g} \in \operatorname{Ham}(\widetilde{M}, \widetilde{\omega})$ of $g$ with respect to $\pi$ is defined to be the time-1 map of the Hamiltonian flow generated by $H_{t} \circ \pi$.

Lemma 2.1 (Existence and uniqueness of admissible lifts). For all $g \in \operatorname{Ham}(M, \omega)$, such an admissible lift $\widetilde{g} \in \operatorname{Ham}(\widetilde{M}, \widetilde{\omega})$ exists and is unique.

Proof. The existence follows from the definition. For the uniqueness, it suffices to show that the admissible lift $\widetilde{g}$ of $g$ is independent of the choice of the Hamiltonian function $H_{t}$.

Note that the choice of $H_{t}$ is equivalent to the choice of the Hamiltonian isotopy $g_{t}$ connecting $i d$ to $g$. For every point $p \in M$, let

$$
\widetilde{e v}_{p}: \pi_{1}(\operatorname{Ham}(M, \omega), i d) \rightarrow \pi_{1}(M, p)
$$

be the map induced by the evaluation map $e v_{p}: \operatorname{Ham}(M, \omega) \rightarrow M$ which takes $g$ to $g(p)$. It follows from Floer theory that for all symplectic manifolds $(M, \omega)$, the induced map $\widetilde{e v}_{p}$ is trivial (see McDuff and Salamon [10, Exercise 11.28, or Seidel [17). This deep result implies that for any two different paths $g_{t}^{1}$ and $g_{t}^{2}$ in $\operatorname{Ham}(M, \omega)$ connecting $i d$ to $g, g_{t}^{1}(p)$ and $g_{t}^{2}(p)$ must be homotopic paths in $M$. Therefore, for every point $\widetilde{p} \in \widetilde{M}$, the image $\widetilde{g}(\widetilde{p})$ of $\widetilde{p}$ under $\widetilde{g}$, being the endpoint of the lift of the path $g_{t}(p)$, is independent of the choice of the Hamiltonian isotopy $g_{t}$. This proves the uniqueness of admissible lifts.

The following theorem is a slight generalization of Theorem 1.13 We consider the symplectic covering map

$$
\pi:\left(\mathbb{T}^{2 n}, 2 \omega\right) \rightarrow\left(\mathbb{T}^{2 n}, \omega\right),(x, y) \mapsto(x, 2 y),
$$

where $(x, y):=\left(x_{1}, \cdots, x_{n} ; y_{1}, \cdots, y_{n}\right)$.

Theorem 2.2. Let $\left(\mathbb{T}^{2 n}, \omega\right)$ be the torus with the standard symplectic form $\omega$, and let $\rho$ be a bi-invariant metric on $\operatorname{Ham}\left(\mathbb{T}^{2 n}, \omega\right)$. Assume that $\exists$ some $\lambda>1$ s.t. $\rho(g) \geqslant \lambda \rho(\widetilde{g})$ for all $g \in \operatorname{Ham}\left(\mathbb{T}^{2 n}, \omega\right)$, where $\widetilde{g}$ is the admissible lift of $g$ with respect to the covering map $\pi$ defined above. Then $\rho$ does not extend to a bi-invariant metric on $\operatorname{Symp}_{0}\left(\mathbb{T}^{2 n}, \omega\right)$.

Remark 2.3. For all $g \in \operatorname{Ham}\left(\mathbb{T}^{2 n}, \omega\right)$, the admissible lift $\widetilde{g}$ of $g$ with respect to $\pi$ is by definition an element in $\operatorname{Ham}\left(\mathbb{T}^{2 n}, 2 \omega\right)$. Since $\operatorname{Ham}\left(\mathbb{T}^{2 n}, 2 \omega\right)=\operatorname{Ham}\left(\mathbb{T}^{2 n}, \omega\right)$ as sets, we can think of $\widetilde{g}$ as an element in $\operatorname{Ham}\left(\mathbb{T}^{2 n}, \omega\right)$. Thus it makes sense to talk about the norm $\rho(\widetilde{g})$ of $\widetilde{g}$.

Remark 2.4. The above theorem also seems to apply to the spectral bi-invariant metric $\gamma$ defined on $\operatorname{Ham}\left(\mathbb{T}^{2 n}, \omega\right)$ such that

$$
\gamma(g):=c([1] ; g)-c\left(\left[\omega^{n}\right] ; g\right)
$$

for all $g \in \operatorname{Ham}\left(\mathbb{T}^{2 n}, \omega\right)$. Here $c([1] ; \cdot)$ and $c\left(\left[\omega^{n}\right] ; \cdot\right)$ denote the section of the action spectrum bundle over $\operatorname{Ham}\left(\mathbb{T}^{2 n}, \omega\right)$ associated to the cohomology classes [1] $\in H^{0}\left(\mathbb{T}^{2 n}\right)$ and $\left[\omega^{n}\right] \in H^{2 n}\left(\mathbb{T}^{2 n}\right)$ respectively. The readers are referred to Schwarz [16] for the case of symplectically aspherical manifolds, and to Oh [1] 
for general symplectic manifolds. We expect to prove that $\gamma$ does not extend to a bi-invariant metric on $\operatorname{Symp}_{0}\left(\mathbb{T}^{2 n}, \omega\right)$ by showing that $\gamma$ satisfies the hypothesis stated in the theorem. This will be studied elsewhere.

We shall mention the following concept of displacement energy which will be used in the final step of the proof of Theorem 2.2. Let $\rho$ be any bi-invariant pseudometric on $\operatorname{Ham}(M, \omega)$. For each subset $U$ of $M$, recall that its displacement energy with respect to $\rho$ is defined to be

$$
\operatorname{de}(U, \rho):=\inf \{\rho(f) \mid f \in \operatorname{Ham}(M, \omega), f(U) \cap U=\emptyset\} .
$$

If the set of such $f$ is empty, we say $\operatorname{de}(U, \rho)=\infty$. The following result is due to Eliashberg and Polterovich [3].

Theorem 2.5 ([3, Theorem 1.3.A]). A bi-invariant pseudo-metric $\rho$ on $\operatorname{Ham}(M, \omega)$ is non-degenerate if and only if de $(U, \rho)>0$ for every non-empty open subset $U$.

We refer to 3] for the proof. One can also find the same argument in the proof of Lemma 3.7 below which is an analogy of the above theorem for bi-invariant metrics on $\operatorname{Symp}_{0}(M, \omega)$.

Proof of Theorem 2.2. Let $\rho$ be any bi-invariant metric on $\operatorname{Ham}\left(\mathbb{T}^{2 n}, \omega\right)$. As in Definition 1.1 for the Hofer metric, we have the concept of bounded and unbounded symplectomorphisms with respect to $\rho$. More precisely, for each $\phi \in \operatorname{Symp}_{0}\left(\mathbb{T}^{2 n}, \omega\right)$, we define

$$
r_{\rho}(\phi):=\sup \left\{\rho([\phi, f]) \mid f \in \operatorname{Ham}\left(\mathbb{T}^{2 n}, \omega\right)\right\},
$$

where $[\phi, f]:=\phi f \phi^{-1} f^{-1}$ is the commutator of $\phi$ and $f$. We say $\phi$ is bounded with respect to $\rho$ if $r_{\rho}(\phi)<\infty$, and unbounded otherwise.

If $\rho$ can extend to a bi-invariant metric on $\operatorname{Symp}_{0}\left(\mathbb{T}^{2 n}, \omega\right)$, still denoted by $\rho$, then given $\phi \in \operatorname{Symp}_{0}\left(\mathbb{T}^{2 n}, \omega\right)$, we have $\rho([\phi, f]) \leqslant 2 \rho(\phi)$ for all $f \in \operatorname{Ham}\left(\mathbb{T}^{2 n}, \omega\right)$. This implies that all symplectomorphisms $\phi \in \operatorname{Symp}_{0}\left(\mathbb{T}^{2 n}, \omega\right)$ are bounded with respect to $\rho$. Thus as in Theorem 1.3, to show $\rho$ does not extend to a bi-invariant metric on $\operatorname{Symp}_{0}\left(\mathbb{T}^{2 n}, \omega\right)$, it suffices to show the existence of some unbounded symplectomorphism with respect to $\rho$ in $\operatorname{Symp}_{0}\left(\mathbb{T}^{2 n}, \omega\right)$.

Let $\phi \in \operatorname{Symp}_{0}\left(\mathbb{T}^{2 n}, \omega\right)$ be the halfway rotation of $\mathbb{T}^{2 n}$ along $x_{1}$-axis, i.e.

$$
\phi\left(x_{1}, \cdots, x_{n} ; y_{1}, \cdots, y_{n}\right)=\left(x_{1}+\frac{1}{2}, \cdots, x_{n} ; y_{1}, \cdots, y_{n}\right) .
$$

We want to show that $\phi$ is unbounded with respect to any bi-invariant metric $\rho$ satisfying the hypothesis of Theorem 2.2. For this, we denote by $V$ the subset of $\mathbb{T}^{2 n}$ defined by $\left\{\left|x_{1}\right|<\frac{1}{4}\right\}$ which is obviously displaced by $\phi$. It is easy to construct a smooth isotopy $f_{s} \in \operatorname{Ham}\left(\mathbb{T}^{2 n}, \omega\right)$ supported in $V$ such that the restriction of $f_{s}$ to the subset $\left\{\left|x_{1}\right|<\frac{1}{8}\right\}$ is defined by

$$
f_{s}\left(x_{1}, \cdots, x_{n} ; y_{1}, \cdots, y_{n}\right)=\left(x_{1}, \cdots, x_{n} ; y_{1}+s, \cdots, y_{n}\right) .
$$

Denote by $g_{s}$ the commutator of $\phi$ and $f_{s}$, i.e. $g_{s}:=\left[\phi, f_{s}\right]=\phi f_{s} \phi^{-1} f_{s}^{-1}$. In order to prove $\phi$ is unbounded with respect to $\rho$, it suffices to show that there exist arbitrarily large $s$ such that $\rho\left(g_{s}\right)$ can be arbitrarily large.

For this, we need to consider the admissible lift $\widetilde{g}_{s}$ of $g_{s}$. Let $m=2^{k}$ for some positive integer $k$. Now consider the covering map

$$
\pi_{m}:\left(\mathbb{T}^{2 n}, m \omega\right) \rightarrow\left(\mathbb{T}^{2 n}, \omega\right),(x, y) \mapsto(x, m y)
$$


Note that $\pi_{m}$ is a symplectic covering map, i.e. $\pi_{m}^{*} \omega=m \omega$ holds. For each $g_{s} \in \operatorname{Ham}\left(\mathbb{T}^{2 n}, \omega\right)$ constructed above, denote by $\widetilde{g}_{s} \in \operatorname{Ham}\left(\mathbb{T}^{2 n}, m \omega\right)$ the unique admissible lift of $g_{s}$ with respect to $\pi_{m}$. Since $\operatorname{Ham}\left(\mathbb{T}^{2 n}, m \omega\right)=\operatorname{Ham}\left(\mathbb{T}^{2 n}, \omega\right)$, as in Remark 2.3, we can think of $\widetilde{g}_{s}$ as an element in $\operatorname{Ham}\left(\mathbb{T}^{2 n}, \omega\right)$. Thus the norm $\rho\left(\widetilde{g}_{s}\right)$ makes sense. Now that $\pi_{m}=\pi^{k}$, where $\pi$ is the covering map in the theorem, the hypothesis on $\rho$ plus an easy induction argument implies that $\rho\left(g_{s}\right) \geqslant \lambda^{k} \rho\left(\widetilde{g}_{s}\right)$. Here $\lambda>1$ is the same constant as in the theorem.

From the construction of $g_{s}=\left[\phi, f_{s}\right]$ and the definition of $\widetilde{g}_{s}$, we can see that if $\frac{m}{4}<s<\frac{m}{2}$, then $\widetilde{g}_{s}$ will displace an open subset $U \subset \mathbb{T}^{2 n}$ defined by

$$
U=\left\{\left(x_{1}, \cdots, x_{n} ; y_{1}, \cdots, y_{n}\right) \in \mathbb{T}^{2 n} \mid-\frac{1}{8}<x_{1}<\frac{1}{8}, 0<y_{1}<\frac{1}{4}\right\} .
$$

Using the definition of the displacement energy, we get $\rho\left(\widetilde{g}_{s}\right) \geqslant \operatorname{de}(U, \rho)>0$. The second inequality holds because of Theorem 2.5. Since $\rho\left(g_{s}\right) \geqslant \lambda^{k} \rho\left(\widetilde{g}_{s}\right)$ holds for some $\lambda>1$, we conclude that $\rho\left(g_{s}\right)$ can be arbitrarily large when $k$ is arbitrarily large. This completes the proof of Theorem 2.2

Remark 2.6. The choice of the symplectic covering map $\pi$ in Theorem 2.2 is a very subtle question. One might expect the same result to hold when choosing different covering maps. This is the case for all

$$
\pi_{m}:\left(\mathbb{T}^{2 n}, m \omega\right) \rightarrow\left(\mathbb{T}^{2 n}, \omega\right),(x, y) \mapsto(x, m y)
$$

with $m \geqslant 2$ a positive integer. The proof goes exactly the same as above, where $m=2$. On the other hand, the argument breaks down if we choose, for instance, the covering map

$$
p:\left(\mathbb{T}^{2 n}, 4 \omega\right) \rightarrow\left(\mathbb{T}^{2 n}, \omega\right),(x, y) \mapsto(2 x, 2 y) .
$$

The reason is as follows.

In view of Theorem 2.5, the displacement energy $\operatorname{de}(U, \rho)$ of any open subset $U \subset \mathbb{T}^{2 n}$ with respect to any bi-invariant metric $\rho$ is always positive. This is sufficient for the proof of Theorem 2.2 since we have been able to show that, by carefully choosing $s$ and $m$, the admissible lift of $g_{s}$ with respect to $\pi_{m}=\pi^{k}$ always displaces some fixed subset $U$ of $\mathbb{T}^{2 n}$ despite the rescaling (enlarging the symplectic form) of the torus. One the other hand, for the covering map $p$, the admissible lift of $g_{s}$ with respect to $p_{m}=p^{k}$ can only be arranged to displace a shrinking portion $U_{m}$ of $\mathbb{T}^{2 n}$. One would still be able to get the same result by carefully analyzing the effect on the capacity of the rescaling process if as for the Hofer metric, the energy-capacity inequality

$$
\operatorname{de}(U, \rho) \geqslant c \cdot \operatorname{capacity}(U, \omega)
$$

holds for our bi-invariant metric $\rho$. However, we do not know if this is true, nor do we have any counter-examples. It would be interesting to have an answer in either direction.

Now back to Theorem 1.13 for $\chi$-invariant Finsler metrics. We begin with a remark on the $\chi$-invariance assumption.

Remark 2.7. We have already mentioned that a $\chi$-invariant norm $\|\cdot\|$ is necessarily $\operatorname{Ham}\left(\mathbb{T}^{2 n}, \omega\right)$-invariant. Hence a $\chi$-invariant Finsler metric $\rho$ must be bi-invariant. Moreover, any $\chi$-invariant norm holds $\|H\|=\|H \circ \pi\|$ for all $H \in \mathcal{A}$, where $\pi: \mathbb{T}^{2 n} \rightarrow \mathbb{T}^{2 n}$ is any covering map of $\mathbb{T}^{2 n}$ over itself. The latter will be crucial for the proof of Theorem 1.13 , 
The following lemma gives a relation between the Finsler norms of a Hamiltonian symplectomorphism and its admissible lift.

Lemma 2.8. Let $\pi:\left(M_{1}, \omega_{1}\right) \rightarrow\left(M_{2}, \omega_{2}\right)$ be a covering map such that $\omega_{1}=\pi^{*} \omega_{2}$. Let $\|\cdot\|_{1},\|\cdot\|_{2}$ be norms on $\mathcal{A}_{1}, \mathcal{A}_{2}$ respectively, such that $\|H \circ \pi\|_{1}=\|H\|_{2}$, and let $\rho_{1}, \rho_{2}$ be the corresponding Finsler pseudo-metrics on $\operatorname{Ham}\left(M_{1}, \omega_{1}\right)$ and $\operatorname{Ham}\left(M_{2}, \omega_{2}\right)$. Then $\rho_{2}\left(g_{2}\right) \geqslant \rho_{1}\left(g_{1}\right)$, where $g_{1} \in \operatorname{Ham}\left(M_{1}, \omega_{1}\right)$ is the admissible lift of $g_{2} \in \operatorname{Ham}\left(M_{2}, \omega_{2}\right)$ with respect to $\pi$.

Proof. By the definition of the admissible lift, we know that if $g_{2} \in \operatorname{Ham}\left(M_{2}, \omega_{2}\right)$ is the time-1 map of the Hamiltonian flow generated by the time-dependent Hamiltonian function $H_{t} \in \mathcal{A}_{2}$, then $g_{1} \in \operatorname{Ham}\left(M_{1}, \omega_{1}\right)$ is the time-1 map of the Hamiltonian flow generated by $H_{t} \circ \pi \in \mathcal{A}_{1}$. Now $\|H\|_{2}=\|H \circ \pi\|_{1}$; by taking the infimum we get $\rho_{2}\left(g_{2}\right) \geqslant \rho_{1}\left(g_{1}\right)$ since the first infimum is taken on a smaller set.

We continue with the following lemma which will deduce Theorem 1.13 from Theorem 2.2.

Lemma 2.9. Let $\rho$ be a Finsler metric on $\operatorname{Ham}\left(\mathbb{T}^{2 n}, \omega\right)$ induced by a $\chi$-invariant norm $\|\cdot\|$. Then $\rho(g) \geqslant 2 \rho(\widetilde{g})$ for all $g \in \operatorname{Ham}\left(\mathbb{T}^{2 n}, \omega\right)$ and the admissible lift $\widetilde{g}$ of $g$ with respect to the symplectic covering map $\pi:\left(\mathbb{T}^{2 n}, 2 \omega\right) \rightarrow\left(\mathbb{T}^{2 n}, \omega\right)$ such that $\pi(x, y)=(x, 2 y)$.

Proof. In view of Remark 2.3, $\operatorname{Ham}\left(\mathbb{T}^{2 n}, 2 \omega\right)=\operatorname{Ham}\left(\mathbb{T}^{2 n}, \omega\right)$, so they share the same Lie algebra $\mathcal{A}$. Let $\rho$ and $\rho_{2}$ be the Finsler metrics on $\operatorname{Ham}\left(\mathbb{T}^{2 n}, \omega\right)$ and $\operatorname{Ham}\left(\mathbb{T}^{2 n}, 2 \omega\right)$ respectively, but both are induced by the same norm $\|\cdot\|$ on $\mathcal{A}$. We claim that $\widetilde{\rho}=2 \rho$. In fact, an element $g \in \operatorname{Ham}\left(\mathbb{T}^{2 n}, \omega\right)$ is the time- 1 map of the Hamiltonian flow generated by some time-dependent Hamiltonian function $H_{t}$ if and only if the same $g$ considered as an element in $\operatorname{Ham}\left(\mathbb{T}^{2 n}, 2 \omega\right)$ is the time-1 map of the Hamiltonian flow generated by $2 H_{t}$. Taking the infimum gives the equality $\rho_{2}(g)=2 \rho(g)$ for all $g$.

On the other hand, the fact that $\|\cdot\|$ is $\chi$-invariant implies $\|H \circ \pi\|=\|H\|$ for all $H \in \mathcal{A}$, where $\pi$ is the covering map in the lemma. Lemma 2.8 implies that $\rho(g) \geqslant \rho_{2}(\widetilde{g})$. Combining this with the previous equality $\rho_{2}=2 \rho$, we obtain $\rho(g) \geqslant 2 \rho(\widetilde{g})$ as desired.

Proof of Theorem 1.13. It follows from the above lemma that any $\chi$-invariant Finsler metric $\rho$ on $\operatorname{Ham}\left(\mathbb{T}^{2 n}, \omega\right)$ must satisfy the hypothesis of Theorem 2.2 with the constant $\lambda=2$. Thus Theorem 1.13 follows.

In the remainder of this section, we try to generalize Theorem 2.2 to other symplectic manifolds such as $\left(\mathbb{T}^{2 n} \times M, \omega \oplus \sigma\right)$. Here $\left(\mathbb{T}^{2 n}, \omega\right)$ is the torus with the standard symplectic form, and $(M, \sigma)$ is any closed symplectic manifold.

Let $\pi:\left(\mathbb{T}^{2 n} \times M, 2 \omega \oplus \sigma\right) \rightarrow\left(\mathbb{T}^{2 n} \times M, \omega \oplus \sigma\right),(x, y, p) \mapsto(x, 2 y, p)$ be a symplectic covering map, i.e. $\pi^{*}(\omega \oplus \sigma)=2 \omega \oplus \sigma$ holds. Thus for every $g \in$ $\operatorname{Ham}\left(\mathbb{T}^{2 n} \times M, \omega \oplus \sigma\right)$, one can define the admissible lift $\widetilde{g} \in \operatorname{Ham}\left(\mathbb{T}^{2 n} \times M, 2 \omega \oplus \sigma\right)$ with respect to $\pi$. Let $\rho$ be a bi-invariant metric on $\operatorname{Ham}\left(\mathbb{T}^{2 n} \times M, \omega \oplus \sigma\right)$. Note that $\operatorname{Ham}\left(\mathbb{T}^{2 n} \times M, 2 \omega \oplus \sigma\right) \neq \operatorname{Ham}\left(\mathbb{T}^{2 n} \times M, \omega \oplus \sigma\right)$; therefore $\rho(\widetilde{g})$ is not always defined for all $\widetilde{g}$. Hence the assumption $\rho(g) \geqslant \lambda \rho(\widetilde{g})$ for all $g$ and $\widetilde{g}$ in Theorem 2.2 makes no sense in this context.

However, both groups $\operatorname{Ham}\left(\mathbb{T}^{2 n} \times M, 2 \omega \oplus \sigma\right)$ and $\operatorname{Ham}\left(\mathbb{T}^{2 n} \times M, \omega \oplus \sigma\right)$ contain the product Hamiltonian diffeomorphisms $f \times g$, where $f \in \operatorname{Ham}\left(\mathbb{T}^{2 n}, \omega\right)=$ 
$\operatorname{Ham}\left(\mathbb{T}^{2 n}, 2 \omega\right)$ and $g \in \operatorname{Ham}(M, \sigma)$, and the admissible lift $\widetilde{f \times g}=\widetilde{f} \times g$ of $f \times g$ is also a product Hamiltonian diffeomorphism. So we can think of $\widetilde{f \times g}$ as an element in $\operatorname{Ham}\left(\mathbb{T}^{2 n} \times M, \omega \oplus \sigma\right)$. Thus the norm $\rho(\widetilde{f \times g})$ does make sense.

The following theorem slightly generalizes Theorem 2.2 .

Theorem 2.10. Let $\rho$ be a bi-invariant metric on $\operatorname{Ham}\left(\mathbb{T}^{2 n} \times M, \omega \oplus \sigma\right)$. Assume that $\exists \lambda>1$ s.t. $\rho(f \times i d) \geqslant \lambda \rho(\widetilde{f \times i d})$ for all $f \times i d \in \operatorname{Ham}\left(\mathbb{T}^{2 n} \times M, \omega \oplus \sigma\right)$ and $\widetilde{f \times i d}$ the admissible lift of $f \times i d$ with respect to $\pi$ described above. Then $\rho$ does not extend to a bi-invariant metric on $\operatorname{Symp}_{0}\left(\mathbb{T}^{2 n} \times M, \omega \oplus \sigma\right)$.

Proof. The proof follows the same lines as that of Theorem 2.2. Let $\phi, f_{s}$ and $g_{s}:=\left[\phi, f_{s}\right]$ be those maps as in the proof of Theorem 2.2. One has to show that $\phi \times i d$ is unbounded with respect to $\rho$ satisfying the hypothesis in this theorem. It suffices to show that $\rho\left(g_{s} \times i d\right)=\rho\left(\left[\phi \times i d, f_{s} \times i d\right]\right)$ can be arbitrarily large. This can easily be achieved by considering the admissible lift of $g_{s} \times i d$ as in Theorem 2.2 ,

Remark 2.11. Theorem 2.2 is a special case of Theorem 2.10 with $M$ being a point. We have already seen that Theorem 2.2 can be applied to $\chi$-invariant Finsler metrics including the Hofer metric. On the other hand, we are not able to find any application of Theorem 2.10 since it is very hard to check its hypothesis. In particular, we do not know if the hypothesis will be satisfied by the Hofer metric. In view of Remark 1.6. however, we already know that the Hofer metric on $\operatorname{Ham}\left(\mathbb{T}^{2 n} \times M, \omega \oplus \sigma\right)$ does not extend bi-invariantly to $\mathrm{Symp}_{0}\left(\mathbb{T}^{2 n} \times M, \omega \oplus \sigma\right)$.

\section{Bi-InVARIANT Metrics ON $\operatorname{Symp}_{0}(M, \omega)$}

In this section, we introduce the concept of $C^{k}$-continuous metrics on $\operatorname{Ham}(M, \omega)$ or $\operatorname{Symp}_{0}(M, \omega)$. One of the main results is Theorem 3.6, which states that there exists no $C^{1}$-continuous bi-invariant metric on $\operatorname{Symp}_{0}\left(\mathbb{T}^{2 n}, \omega\right)$. We also construct two families of bi-invariant metrics on $\operatorname{Symp}_{0}(M, \omega)$ and study their topological properties.

3.1. $C^{k}$-continuous metrics. We begin with the definition of $C^{k}$-continuous biinvariant metrics. Here we use the term $\rho$-topology for the topology induced by the metric $\rho$.

Definition 3.1. Let $k=0$ or 1 , and let $G$ be either $\operatorname{Ham}(M, \omega)$ or $\operatorname{Symp}_{0}(M, \omega)$. A bi-invariant metric $\rho$ on $G$ is said to be $C^{k}$-continuous if the $C^{k}$-topology on $G$ is finer than $\rho$-topology, or equivalently, if the identity map on $G$

$$
\operatorname{Id}_{G}:\left(G, C^{k} \text {-topology }\right) \rightarrow(G, \rho \text {-topology })
$$

is a continuous map.

The following proposition is trivial from the definition of the Hofer metric.

Proposition 3.2. For all $(M, \omega)$ the Hofer metric on $\operatorname{Ham}(M, \omega)$ is $C^{1}$-continuous.

In general, the Hofer metric is not $C^{0}$-continuous. This seems obvious, since the definition of the Hofer length of a smooth path uses the derivative of the path. To produce a counter-example, however, we are forced to use the following deep result by Polterovich [15]. 
Theorem 3.3 (15, Theorem 1.A]). Let $L \subset S^{2}$ be an equator of the standard 2 -sphere $S^{2}$ with area form $\omega$. Let $f$ be a Hamiltonian diffeomorphism of $S^{2}$ generated by a Hamiltonian function $F \in \mathcal{A}$. Assume that for some positive number $c$, $F(x, t) \geqslant c$ for all $x \in L$ and $t \in S^{1}$. Then the Hofer norm $\rho(f) \geqslant c$.

Proposition 3.4. The Hofer metric on $\operatorname{Ham}\left(S^{2}, \omega\right)$ is not $C^{0}$-continuous.

Proof. For any $c>0$, take a sequence of autonomous Hamiltonian functions $F_{n} \in \mathcal{A}$ such that $F_{n}=c$ except for a small disc $B_{n} \subset S^{2}$ whose diameter goes to 0 as $n$ goes to infinity. Let $f_{n} \in \operatorname{Ham}\left(S^{2}, \omega\right)$ be the Hamiltonian diffeomorphisms generated by $F_{n}$. It follows from Theorem 3.3 that the Hofer norm $\rho\left(f_{n}\right) \geqslant c$. On the other hand, the $C^{0}$-limit of the sequence $f_{n}$ is the identity map $i d \in \operatorname{Ham}\left(S^{2}, \omega\right)$ with $\rho(i d)=0$. This shows $\rho$ is not $C^{0}$-continuous.

Remark 3.5. In view of [14, Theorem 7.2.C, the above construction also works for closed surfaces $\Sigma$ of genus $>0$ where any non-contractible closed curve $L$ in $\Sigma$ plays the same role as the equator in $S^{2}$. It also holds for $S^{2} \times S^{2}$ with the split symplectic form $\omega \oplus \omega$ and $\mathbb{C} P^{n}$ endowed with the Fubini-Study form, using the Calabi quasimorphism constructed by Entov and Polterovich on the Hamiltonian group of these manifolds (cf. Remark 1.10 of [4]).

We see from Proposition 3.2 that $C^{1}$-continuous bi-invariant metrics such as the Hofer metric always exist on $\operatorname{Ham}(M, \omega)$ for all $(M, \omega)$. However, this is not true in general for $\operatorname{Symp}_{0}(M, \omega)$. In particular, we have

Theorem 3.6. There is no $C^{1}$-continuous bi-invariant metric on $\operatorname{Symp}_{0}\left(\mathbb{T}^{2 n}, \omega\right)$.

To prove this theorem, we need the following lemma which is analogous to Theorem 2.5 (Theorem 1.3.A of [3]). Let $\rho$ be any bi-invariant pseudo-metric on $\operatorname{Symp}_{0}(M, \omega)$. For each subset $U$ of $M$, we define its symplectic displacement energy with respect to $\rho$ :

$$
d e^{s}(U, \rho):=\inf \left\{\rho(\phi) \mid \phi \in \operatorname{Symp}_{0}(M, \omega), \phi(U) \cap U=\emptyset\right\} .
$$

If the set of such $\phi$ is empty, we say $d e^{s}(U, \rho)=\infty$.

Lemma 3.7. A bi-invariant pseudo-metric $\rho$ on $\operatorname{Symp}_{0}(M, \omega)$ is non-degenerate if and only if $d e^{s}(U, \rho)>0$ for every non-empty open subset $U$.

Proof. Our argument follows along the same lines as that of Theorem 1.3.A in 3. Assume $d e^{s}(U, \rho)>0$ for all non-empty open subsets $U$. Since each nonidentity map $\phi \in \operatorname{Symp}_{0}(M, \omega)$ must displace some small ball $U \subset M$, we get that $\rho(\phi) \geqslant d e^{s}(U, \rho)>0$. For the converse, note that for any non-empty open set $U \subset M$, there exist $\phi, \psi \in \operatorname{Symp}_{0}(M, \omega)$ supported in $U$ such that $[\phi, \psi] \neq i d$. Since $\rho$ is non-degenerate, $\rho([\phi, \psi])>0$. To complete our argument, it suffices to prove the following claim.

Claim. Let $U \subset M$ be a non-empty open subset. For all $\phi, \psi \in \operatorname{Symp}_{0}(M, \omega)$ with $\operatorname{supp}(\phi) \subset U$ and $\operatorname{supp}(\psi) \subset U$, we have $d e^{s}(U, \rho) \geqslant \frac{1}{4} \rho([\phi, \psi])$.

For the argument of the claim, assume there exists $\eta \in \operatorname{Symp}_{0}(M, \omega)$ such that $\eta(U) \cap U=\emptyset$ (if such an $\eta$ does not exist we are done, because $d e^{s}(U, \rho)=\infty$ ). Set $\theta:=[\phi, \eta]=\phi \eta \phi^{-1} \eta^{-1}$. Using the fact that $\eta$ displaces $U$ and that $\phi, \psi$ are supported in $U$, one can easily verify that $[\phi, \psi]=[\theta, \psi]$. Therefore we get

$$
\rho([\phi, \psi])=\rho([\theta, \psi]) \leqslant 2 \rho(\theta)=2 \rho([\phi, \eta]) \leqslant 4 \rho(\eta) .
$$


Here we have used the bi-invariance of $\rho$ and the triangle inequality. Since this holds for all $\eta \in \operatorname{Symp}_{0}(M, \omega)$ with $\eta(U) \cap U=\emptyset$, we obtain $d e^{s}(U, \rho) \geqslant \frac{1}{4} \rho([\phi, \psi])$ by taking the infimum over all such $\eta$ 's.

Proof of Theorem 3.6. Let

$$
\phi_{\alpha}: \mathbb{T}^{2 n} \rightarrow \mathbb{T}^{2 n},\left(x_{1}, \cdots, x_{n} ; y_{1}, \cdots, y_{n}\right) \mapsto\left(x_{1}+\alpha, \cdots, x_{n} ; y_{1}, \cdots, y_{n}\right) .
$$

We have the following

Claim. For each $0<\alpha<\frac{1}{8}$, there exists $\psi_{\alpha} \in \operatorname{Symp}_{0}\left(\mathbb{T}^{2 n}, \omega\right)$ such that the conjugate $\psi_{\alpha} \phi_{\alpha} \psi_{\alpha}^{-1}$ of $\phi_{\alpha}$ will displace an open set $U$ of $\mathbb{T}^{2 n}$ which is independent of $\alpha$.

Assume the Claim to be true for the moment. For any bi-invariant metric $\rho$ on $\operatorname{Symp}_{0}\left(\mathbb{T}^{2 n}, \omega\right)$, we have $\rho\left(\phi_{\alpha}\right)=\rho\left(\psi_{\alpha} \phi_{\alpha} \psi_{\alpha}^{-1}\right) \geqslant d e(U, \rho)$, where the last term $d e(U, \rho)$ is a fixed positive number by Lemma 3.7. Since the $C^{1}$-limit of $\phi_{\alpha}$ as $\alpha$ approaches 0 is the identity map $i d \in \operatorname{Symp}_{0}\left(\mathbb{T}^{2 n}, \omega\right)$, we conclude that $\rho$ is not $C^{1}$-continuous.

It suffices to prove the Claim by direct construction. For each $0<\alpha<\frac{1}{8}$, let $h_{\alpha}: S^{1} \rightarrow \mathbb{R}$ be a smooth function such that $h_{\alpha}(x+\alpha)-h_{\alpha}(x)=\frac{1}{2}$ for $\frac{1}{4}<x<\frac{3}{4}$. Define $\psi_{\alpha}: \mathbb{T}^{2 n} \rightarrow \mathbb{T}^{2 n}$ such that

$$
\psi_{\alpha}\left(x_{1}, \cdots, x_{n} ; y_{1}, \cdots, y_{n}\right)=\left(x_{1}, \cdots, x_{n} ; y_{1}+h_{\alpha}\left(x_{1}\right), \cdots, y_{n}\right) .
$$

Note that $\psi_{\alpha} \in \operatorname{Symp}_{0}\left(\mathbb{T}^{2 n}, \omega\right)$. Now $\psi_{\alpha} \phi_{\alpha} \psi_{\alpha}^{-1}$ maps $\left(x_{1}, \cdots, x_{n} ; y_{1}, \cdots, y_{n}\right)$ to $\left(x_{1}+\alpha, \cdots, x_{n} ; y_{1}+h_{\alpha}\left(x_{1}+\alpha\right)-h_{\alpha}\left(x_{1}\right), \cdots, y_{n}\right)$, which displaces the open set $U \subset \mathbb{T}^{2 n}$ defined by

$$
U=\left\{\left(x_{1}, \cdots, x_{n} ; y_{1}, \cdots, y_{n}\right) \in \mathbb{T}^{2 n} \mid \frac{1}{4}<x_{1}<\frac{3}{4}, 0<y_{1}<\frac{1}{4}\right\} .
$$

This completes the proof of the Claim, hence the theorem.

Remark 3.8. Theorem 3.6 can be generalized to $\left(\mathbb{T}^{2 n} \times M, \omega \oplus \sigma\right)$. That is, there exists no $C^{1}$-continuous bi-invariant metric on $\operatorname{Symp}_{0}\left(\mathbb{T}^{2 n} \times M, \omega \oplus \sigma\right)$, where $\left(\mathbb{T}^{2 n}, \omega\right)$ is the standard torus and $(M, \sigma)$ is any closed symplectic manifold. This is true since one can show, as in the proof of Theorem 3.6. that the conjugate of $\phi_{\alpha} \times i d$ will displace some fixed subset $U \times M$ of $\mathbb{T}^{2 n} \times M$. Here $\phi_{\alpha}$ denote the same rotation maps of $\mathbb{T}^{2 n}$ as above.

3.2. Bi-invariant metrics on $\operatorname{Symp}_{0}(M, \omega)$. In this subsection, we present two families of bi-invariant metrics on $\operatorname{Symp}_{0}(M, \omega)$ and discuss their topological properties. In both constructions, $(M, \omega)$ is a closed symplectic manifold and $\rho$ is the Hofer norm on $\operatorname{Ham}(M, \omega)$. The first construction is due to Lalonde and Polterovich [8]. For every positive number $a$, we define $r_{a}: \operatorname{Symp}_{0}(M, \omega) \rightarrow \mathbb{R}$ such that for all $\phi \in \operatorname{Symp}_{0}(M, \omega)$,

$$
r_{a}(\phi):=\sup \{\rho([\phi, f]) \mid f \in \operatorname{Ham}(M, \omega), \rho(f) \leqslant a\},
$$

where $[\phi, f]:=\phi f \phi^{-1} f^{-1}$ is the commutator of $\phi$ and $f$.

Proposition 3.9 ([8, Prop 1.2.A]). For every $a \in(0, \infty)$, the function $r_{a}$ is a bi-invariant norm on $\operatorname{Symp}_{0}(M, \omega)$. 
For the second construction, let $K>0$. Define $\rho_{K}: \operatorname{Symp}_{0}(M, \omega) \rightarrow \mathbb{R}$ such that for all $\phi \in \operatorname{Symp}_{0}(M, \omega)$,

$$
\rho_{K}(\phi):= \begin{cases}\min (\rho(\phi), K), & \text { if } \phi \in \operatorname{Ham}(M, \omega), \\ K, & \text { otherwise. }\end{cases}
$$

Proposition 3.10. For every $K \in(0, \infty)$, the function $\rho_{K}$ is a bi-invariant norm on $\operatorname{Symp}_{0}(M, \omega)$.

The proofs of both propositions above are straightforward and therefore omitted.

Remark 3.11. $r_{a}$ and $\rho_{K}$ restrict to bi-invariant norms on $\operatorname{Ham}(M, \omega)$. One can think of $r_{a}$ and $\rho_{K}$ to be bi-invariant extensions of their corresponding norms on $\operatorname{Ham}(M, \omega)$. Note that the diameter of $\operatorname{Ham}(M, \omega)$ with respect to these metrics is finite, so one can always extend them bi-invariantly to $\operatorname{Symp}_{0}(M, \omega)$ by giving a sufficiently large constant value for all non-Hamiltonian symplectomorphisms. Compare this with Question 1.14 .

For the properties concerning these metrics, first we shall see that for any $(M, \omega)$ such that $\operatorname{Symp}_{0}(M, \omega)$ is not identical to $\operatorname{Ham}(M, \omega), \rho_{K}$ is not $C^{1}$-continuous on $\operatorname{Symp}_{0}(M, \omega)$. This is true since $\rho_{K}(\phi)=K$ for all non-Hamiltonian symplectomorphisms $\phi$ and $\rho_{K}(i d)=0$. We do not know as much for bi-invariant metrics $r_{a}$. However, we do know that $r_{a}$ is not $C^{1}$-continuous on $\operatorname{Symp}_{0}\left(\mathbb{T}^{2 n}, \omega\right)$ for the standard torus $\left(\mathbb{T}^{2 n}, \omega\right)$ in view of Theorem 3.6. This can also be proved directly, using the fact that $r_{a}(\phi)=2 a$ for every non-identity rotation $\phi$ and $r_{a}(i d)=0$. On the other hand, the restrictions of $r_{a}$ and $\rho_{K}$ to $\operatorname{Ham}(M, \omega)$ are $C^{1}$-continuous, since both are bounded from above by the Hofer norm. More precisely, we have $r_{a}(f) \leqslant 2 \rho(f)$ and $r_{a}(f) \leqslant 2 \rho(f)$ for all $f \in \operatorname{Ham}(M, \omega)$. Since the Hofer metric is $C^{1}$-continuous according to Proposition [3.2, $r_{a}$ and $\rho_{K}$ are also $C^{1}$-continuous.

For bi-invariant metrics $\rho_{K}$, we also have the following easy result.

Proposition 3.12. For every $K>0$, the identity component of $\operatorname{Symp}_{0}(M, \omega)$ with respect to the $\rho_{K}$-topology is $\operatorname{Ham}(M, \omega)$.

Proof. For all $f \in \operatorname{Ham}(M, \omega)$ and $\phi \notin \operatorname{Ham}(M, \omega)$, we have the distance $d(f, \phi)=$ $\rho\left(\phi f^{-1}\right)=K$ since $\phi f^{-1} \notin \operatorname{Ham}(M, \omega)$. On the other hand, $\operatorname{Ham}(M, \omega)$ is obviously path-connected with respect to $\rho_{K}$-topology. The proposition follows immediately.

This easy observation leads us to the following question. We content ourselves with formulating the question only in terms of the standard torus $\left(\mathbb{T}^{2 n}, \omega\right)$.

Question 3.13. Is $\operatorname{Ham}\left(\mathbb{T}^{2 n}, \omega\right)$ the identity component of $\operatorname{Symp}_{0}\left(\mathbb{T}^{2 n}, \omega\right)$ with respect to the $r_{a}$-topology? Is it true for all bi-invariant metrics on $\operatorname{Symp}_{0}\left(\mathbb{T}^{2 n}, \omega\right)$ ?

Proposition 3.12 gives a positive answer to the above question for bi-invariant metrics $\rho_{K}$. For bi-invariant metrics $r_{a}$, as a partial answer, we have the following theorem which states that an $r_{a}$-continuous smooth isotopy in $\operatorname{Symp}_{0}\left(\mathbb{T}^{2 n}, \omega\right)$ must lie entirely in $\operatorname{Ham}\left(\mathbb{T}^{2 n}, \omega\right)$.

Theorem 3.14. Let $\gamma:[0,1] \rightarrow \operatorname{Symp}_{0}\left(\mathbb{T}^{2 n}, \omega\right)$ be a smooth isotopy, i.e. a $C^{1}$ continuous path starting from id. Then $\gamma$ is $r_{a}$-continuous if and only if it is a smooth isotopy in $\operatorname{Ham}\left(\mathbb{T}^{2 n}, \omega\right)$. 
Proof. Let $\gamma$ be a smooth isotopy in $\operatorname{Ham}\left(\mathbb{T}^{2 n}, \omega\right)$, i.e. $\gamma$ is a $C^{1}$-continuous path with $\gamma_{0}=i d$. We have pointed out above that the bi-invariant metric $r_{a}$, when restricted to $\operatorname{Ham}\left(\mathbb{T}^{2 n}, \omega\right)$, is $C^{1}$-continuous in the sense of Definition 3.1. Thus the fact that $\gamma$ is a $C^{1}$-continuous path implies that it is also $r_{a}$-continuous.

On the other hand, we have to show that if there exists some $t_{0} \in[0,1]$ such that $\gamma_{t_{0}} \notin \operatorname{Ham}\left(\mathbb{T}^{2 n}, \omega\right)$, then $\gamma$ is not $r_{a}$-continuous. For each $t \in[0,1]$, we have the unique decomposition $\gamma_{t}=\phi_{t} \circ f_{t}$, where $\phi_{t}$ is the unique rotation of the torus such that $f_{t}=\phi_{t}^{-1} \circ \gamma_{t}$ is in $\operatorname{Ham}\left(\mathbb{T}^{2 n}, \omega\right)$. Note that $\phi_{0}=f_{0}=i d$, and the assumption $\gamma_{t_{0}} \notin \operatorname{Ham}\left(\mathbb{T}^{2 n}, \omega\right)$ for some $t_{0}$ implies $\phi_{t_{0}} \neq i d$. Now $\gamma$ is a $C^{1}$-continuous path, and so are the paths $\phi$ and $f$. Since $r_{a}$ is $C^{1}$-continuous when restricted to $\operatorname{Ham}\left(\mathbb{T}^{2 n}, \omega\right)$, the fact that $f$ is a $C^{1}$-continuous path in $\operatorname{Ham}\left(\mathbb{T}^{2 n}, \omega\right)$ implies that $f$ is also $r_{a^{-}}$ continuous. If the path $\gamma$ were $r_{a}$-continuous, it would imply that the path $\phi$ is also $r_{a}$-continuous. However, as we already pointed out before, for each $a, r_{a}$ only assumes two values on rotations, i.e. $r_{a}(i d)=0$, and $r_{a}(\psi)=2 a$ for all non-identity rotations $\psi$. Since we have $\phi_{0}=i d$, and $\phi_{t_{0}} \neq i d$ for some $t_{0}$, it is not possible for the path $\phi$ to be $r_{a}$-continuous, which is the contradiction. The proof is therefore completed.

Remark 3.15. The proof of Theorem 3.14 implies that for $\left(\mathbb{T}^{2 n}, \omega\right)$, the distance between Hamiltonian symplectomorphisms and non-Hamiltonian symplectomorphisms with respect to $r_{a}$ is bounded away from 0 by some constant if the two elements are $C^{1}$-close. If this remains true when they are not $C^{1}$-close, then the answer to Question 3.13 would be positive for bi-invariant metrics $r_{a}$. However, we do not know this yet at this time.

\section{ACKNOWLEDGEMENTS}

This work is part of the author's Ph.D. thesis, being carried out under the supervision of Professor Dusa McDuff at Stony Brook University. He would like to thank Professor McDuff for her great guidance and continual support.

\section{REFERENCES}

[1] A. Banyaga, Sur la structure du groupe des difféomorphisms qui préservent une forme symplectique, Comment. Math. Helv. 53, 174-227 (1978) MR490874(80c:58005)

[2] A. Banyaga and P. Donato, Lengths of contact isotopies and extensions of the Hofer metric, Annals of Global Analysis and Geometry 30, 299-312 (2006) MR2256527 (2007i:53097)

[3] Y. Eliashberg and L. Polterovich, Bi-invariant metrics on the group of Hamiltonian diffeomorphisms, Internat. J. Math. 4, 727-738 (1993) MR1245350 (94i:58029)

[4] M. Entov and L. Polterovich, Calabi quasimorphism and quantum homology, Internat. Math. Research Notices 30, 1635-1676 (2003) MR.1979584(2004e:53131)

[5] Z. Han, The bounded isometry conjecture for the Kodaira-Thurston manifold and the 4-torus, to appear in Israel J. Math., arXiv:0705.0762

[6] H. Hofer, On the topological properties of the symplectic maps, Comment. Math. Helv. 68, 25-38 (1990) MR.1059642 (91h:58042)

[7] F. Lalonde and D. McDuff, The geometry of symplectic energy, Ann. Math. 141, 349-371 (1995) MR.1324138 (96a:58089)

[8] F. Lalonde and L. Polterovich, Symplectic diffeomorphisms as isometries of Hofer's norm, Topology 36(3), 711-727 (1997) MR1422431 (97g:58021)

[9] F. Lalonde and C. Pestieau, Stabilisation of symplectic inequalities and applications, Amer. Math. Soc. Transl. (2) 196, 63-72 (1999) MR.1736214(2001g:53152)

[10] D. McDuff and D. Salamon, Introduction to Symplectic Topology, 2nd edition (1998) OUP, Oxford, United Kingdom MR:1698616 (2000g:53098) 
[11] Y.-G. Oh, Chain level Floer theory and Hofer's geometry of the Hamiltonian diffeomorphism group, Asian J. Math. 6, 799-830 (2002) MR1958084 (2003m:53157)

[12] Y. Ostrover and R. Wagner, On the extremality of Hofer's metric on the group of Hamiltonian diffeomorphisms, Internat. Math. Research Notices 35, 2123-2142 (2005) MR2181789 (2006k:58008)

[13] L. Polterovich, Symplectic displacement energy for Lagrangian submanifolds, Ergodic Theory and Dynamical Systems 13, 357-367 (1993) MR1235478 (94h:58081)

[14] L. Polterovich, The geometry of the group of symplectic diffeomorphisms, Lectures in Math, ETH, Birkhäuser (2001) MR1826128 (2002g:53157)

[15] L. Polterovich, Hofer's diameter and Lagrangian intersections, Internat. Math. Research Notices 4, 217-223 (1998) MR1609620 (99b:58037)

[16] M. Schwarz, On the action spectrum for closed symplectically aspherical manifolds, Pacific Journal of Math. No. 2, Vol. 193, 419-461 (2000) MR.1755825 (2001c:53113)

[17] P. Seidel, $\pi_{m}$ of symplectic automorphism groups and invertibles in quantum homology rings, Geom. Funct. Anal. 7, 1046-1095 (1997) MR1487754(99b:57068)

Department of Mathematics and Statistics, University of Massachusetts, Amherst, Amherst, MassachusetTs 01003-9305

E-mail address: han@math.umass.edu 